\title{
High-Efficiency Resonant Cavity Quadrupole Moment Monitor
}

\author{
N. Barov ${ }^{1}$, C.D. Nantista ${ }^{2}$, and R.H. Miller ${ }^{2}$, J.S. Kim ${ }^{1}$ \\ ${ }^{1}$ FAR-TECH, Inc., 10350 Science Ctr. Dr, Bldg. 14, Ste. 150, San Diego, CA 92121 \\ ${ }^{2}$ Stanford Linear Accelerator Center, Menlo Park, CA
}

\begin{abstract}
Measurement of the beam quadrupole moment at several locations can be used to reconstruct the beam envelope and emittance parameters. The measurements can be performed in a non-intercepting way using a set of quadrupole-mode cavities. We present a cavity design with an optimized quadrupole moment shunt impedance. The cavity properties can be characterized using a wire test method to insure symmetry about the central axis, and alignment to nearby position sensing cavities. The design and characterization of the prototype structure is discussed.
\end{abstract}

Keywords: BPM cavity emittance beam quadrupole moment

PACS: 29.27.Fh,29.17.+w

\section{INTRODUCTION}

Resonant cavity-based beam position monitors (BPM's) have been developed to provide extremely accurate measurements of a charged particle beam's position, to the level of $10 \mathrm{~nm}$. These devices measure the first moment of the beam distribution, $\langle x\rangle$ or $\langle y\rangle$ in the transverse coordinates. The same method can be used with cavities operating with a higher harmonic to measure the second-order, or quadrupole moment of a beam. The second-order moment measures either $\left\langle x^{2}-y^{2}\right\rangle$, where the angle brackets indicate averging over the beam population. A technique for emittance measurement using quadrupole moment information from stripline BPM's has been suggested by Miller [1].

The beam quadrupole moment can be measured, for example, with a rectangular pillbox cavity operating in the $\mathrm{TM}_{220}$ mode[2,3]. The cavity can measure the normal quadrupole moment $\left\langle x^{2}-y^{2}\right\rangle$, or the skew quadrupole moment $\langle x y\rangle$, depending its alignment in the azimuthal direction ( 0 or 45 degrees). Evaluating this expression in the case of a beam with transverse sizes $\sigma_{x}$ and $\sigma_{y}$, the output power will be proportional to,

$$
P_{\text {out }} \propto\left(\langle x\rangle^{2}-\langle y\rangle^{2}+\sigma_{x}^{2}-\sigma_{y}^{2}\right)^{2} .
$$

In order to extract information about the beam sizes, one needs to perform a separate dipole measurement as close as possible to the quadrupole cavity, in order to subtract the influence of $\langle x\rangle$ and $\langle y\rangle$ in the expression.

FAR-TECH, Inc., in collaboration with SLAC is developing an integrated beam monitor that can perform the quadrupole and dipole moment measurements. The

Contributed to 12th Advanced Accelerator Concepts Workshop (AAC 2006),

07/10/2006--7/15/2006, Lake Geneva, Wisconsin

Work supported in part by US Department of Energy contract DE-AC02-76SF00515 
geometry of the quadrupole cells has been enhanced to optimize the structure shunt impedance, as will be discussed below.

A collection of six monitors can be installed in a beamline to perform an online non-intercepting measurement of the emittance and other beam parameters such as the beam size. This capability can decrease the amount of time spent in tuning up the beamline. It is also a candidate for inclusion into feedback systems that continually or periodically optimize beam conditions during an experiment. The non-intercepting nature of the technique allows it to be used for beam measurements on very average power beams.

The quadrupole moment measurements can also be used to measure $x-y$ beam coupling by measuring the skew quadrupole moment $\langle x y\rangle$. Additionally, the monitor can be placed in a dispersive region to provide non-invasive information about beam energy spread.

\section{HIGH-EFFICIENCY QUADRUPOLE-MODE CAVITIES}

The shunt impedance of a quadrupole-mode cavity can be greatly enhanced by adding a set of internal posts as shown in Fig. 1. A similar idea was used to increase the shunt impedance of a dipole-mode deflection cavity [4]. In addition to increasing the shunt impedance, this geometry can also be used to tune the remaining cavity modes to be well-separated in frequency from the quadrupole mode. The enhancement of the shunt impedance is shown in Fig. 2. Holding the beam parameters constant, the energy deposited into this type of a cavity will be an order of magnitude higher than for a plain pillbox-type cavity. A US patent on this cavity design has been applied for.

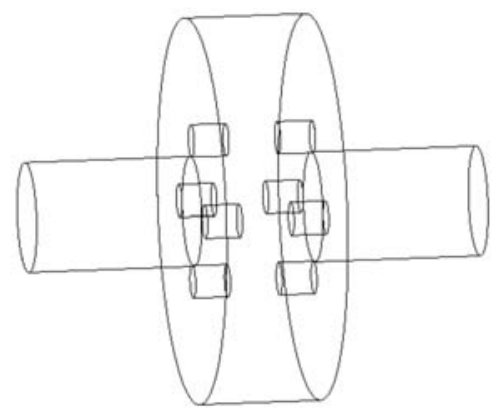

FIGURE 1. A quadrupole mode cavity with internal posts that form four capacitive gaps. The four gaps are located near the maxima in the electric field. 


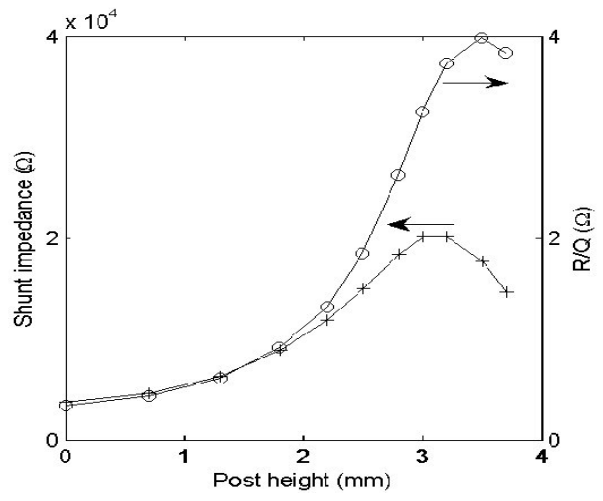

FIGURE 2. The shunt impedance measured at $2 \mathrm{~mm}$ offset is enhanced by a factor of five, and R/Q is enhanced by a factor of $\sim 11$ relative to a cavity without posts. In all cases the cavity outer wall was adjusted to maintain the same resonant frequency.

\section{DETERMINATION OF MEASUREMENT ERRORS}

The method of measuring emittance with six or more quadrupole moment monitors located near the magnets of a FODO lattice was developed by Miller, et al. [1]. This experiment used existing stripline BPM's to interpret the quadrupole moment of the beam at each location. The quadrupole moments at the $i^{\text {th }}$ location are proportional to components of the sigma matrix $\sigma_{i, 11}-\sigma_{i, 33}$. This matrix can be related to the initial sigma matrix by use of the transport matrix, $R_{i}$ between the two locations according to the formula $\sigma_{i}=R_{i} \sigma_{0} R_{i}^{T}$. Assuming that there is no beam $x-y$ coupling, this yields a system of six equations where the unknowns are the six independent components of the transverse coordinates in the sigma matrix.

The six equations are solved by matrix inversion. However, in some cases, the matrix is singular or nearly singular, which causes the error bars associated with the initial beam sigma matrix to blow up in magnitude. The error analysis aspects of a set of beam quadrupole measurements are discussed in references [1,5]. In particular, any pair of measurements that are related by an imaging condition due to a betatron phase advance of a multiple of 180 degrees are not independent, and thus result in a noninvertible matrix.

The best possible strategy for decreasing the error bars is to have a nearly parallel beam at each measurement location, but with a large beam aspect ratio $\left(\sigma_{x}^{2} \ll \sigma_{y}^{2}\right.$, or $\sigma_{x}^{2} \gg \sigma_{y}^{2}$ ). In this way, the influence on the quadrupole moment due to the lesser quantity will be negligible. The initial beam parameters can then be reconstructed by making three such measurements in each dimension separated by 120 degrees of phase advance. In the case of a FODO lattice, the ratio $\sigma_{x}$ to $\sigma_{y}$ can be around 2:1, which therefore reduces the error bar magnitudes. In the measurement by Miller, the lattice phase advance per cell was 72 degrees, which decouples the measurements performed at each measurement location. 


\section{WIRE TESTS}

Although a carefully performed beam quadruple measurement can measure beam sizes below 10 microns (where one dimension is 10 microns and the other is negligible), irregularities in the cavity geometry can easily decrease that accuracy. Specifically, if the height of each post has some random error, the cavity electric field profile will undergo a baseline shift, so that the cavity will measure $<x^{2}-y^{2}>+K$, where $K$ is a constant. Very small changes in the geometry can result in a large $K$, so that a round on-axis beam will generate a signal indicating a beam of 100 's of microns, or even $1 \mathrm{~mm}$ in size. Although the measurement offset can be calibrated, such a large signal is undesirable because it can saturate the receiver electronics. The solution is to conduct a series of measurements using a wire test technique in order to tune the cavity to minimize the offset. The wire test stand for conducting such measurements was originally set up by N. Baboi for the purpose of measuring deflection wakes in the NLC structures [6]. In these measurements, a wire is placed at the device axis so that it forms two co-axial transmission lines at the upstream and downstream beam pipes.

Previous wire test measurements used for mapping deflection wakes in accelerator structures have examined the transmission and reflection characteristics between the two co-axial ports. However, for our case, this does not give a good enough accuracy, and it is necessary to interpret the signal from the device output waveguide. This signal is harder to interpret because it includes a component from a nearby broad resonance. The test setup was simulated with HFSS, and these simulations predict what the network analyzer S parameters should look like for a given amount of mode distortion and wire displacement.

A special cavity was constructed to make an initial test. This cavity resonates at 14.5 GHz and has set screws that can be used to adjust the mode offset. Preliminary measurements on the test stand were successful in identifying the resonances. A more thorough study of the mapping and cancellation of the quadrupole mode offset is ongoing.

\section{STRUCTURE FABRICATION}

The integrated monitor including a two-cell quadrupole cavity and two single-cell dipole BPM cavities is envisioned to look like the image in Fig. 3. The same figure also shows the copper pieces for the quadrupole section. The cavities have internal rods for shunt impedance enhancement, as discussed in a previous section. These pieces have been RF tested and resonate close to the $11.384 \mathrm{GHz}$ target frequency. 

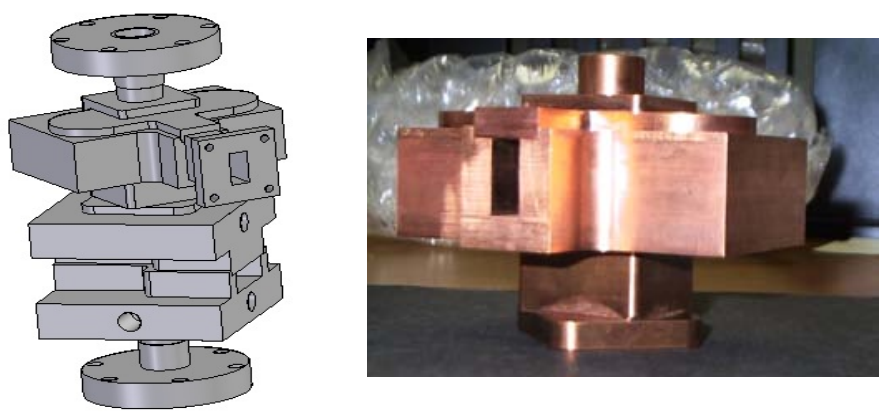

FIGURE 3. The integrated monitor as designed with a CAD program (on the left), and the copper pieces for the quadrupole cells (on the right). In the picture on the left, the bottom half of the structure is the dipole mode cavities.

This design has achieved a reduction in the number of pieces required to construct the monitor. The main body of the monitor, excluding window hardware and flanges, consists of six pieces. This is a simplification from earlier designs which featured a waveguide network with three hybrid tees in order to isolate the quadrupole mode. In the present design each waveguide couples to both quadrupole cavities in a way that singles out the $\pi$-mode, and rejects the 0 -mode. The waveguide network also rejects the monopole and dipole modes.

The quadrupole structure has been measured on a network analyzer and resonates at $11.368 \mathrm{GHz}$, which can easily be tuned closer to the $11.384 \mathrm{GHz}$ target frequency, while anticipating the extra .001 " material for the braze shim. The two cells are tuned within $2 \mathrm{MHz}$ of each other. Due to the strong coupling between cells, this leads to only a slight field imbalance between the cells. We have measured the effect of coupling to the 0 -mode as a function of this imbalance. The present state of tune leads to very little coupling to this mode. Also, the 0 -mode is $>100 \mathrm{MHz}$ away in frequency, and can easily be filtered out.

\section{ACKNOWLEDGMENTS}

We wish to thank Jim Lewandowski of SLAC for his assistance with the wire test measurements.

This work was supported by the US DOE Grant No. DE-FG02-03ER83658.

\section{REFERENCES}

1. R.H. Miller, et al, $12^{\text {th }}$ Intl. Conf. on High-Energy Acccelerators, Fermilab, (1983). (also available as SLACPUB-3186).

2. D.H. Whittum and Y.K. Kolomensky, Rev. Sci. Instr. 70, 5, 2300 (1999).

3. J.S. Kim, C.D. Nantista, R.H. Miller, A.W. Weidemann, Rev. Sci. Instr. 76125109 (2005).

4. C. Leemann and C.G. Yao, Proceedings of the 1990 LINAC Conference, p. 232, (1990).

5. S. Russel, Nucl. Inst. and Meth. A 430, 498 (1999).

6. N. Baboi, et al. SLAC-PUB-9807. 